Abstract IDDF2020-ABS-0103 Table 1 Objective responses and disease control rates between two groups per the RECIST v1.1 and imRECIST.

\begin{tabular}{|c|c|c|c|c|c|c|c|c|}
\hline & \multicolumn{4}{|c|}{ RECIST v1.1 } & \multicolumn{4}{|c|}{ imRECIST } \\
\hline & OR & NR & DC & PD & OR & NR & DC & PD \\
\hline PVTT group $(n=16)$ & & $16(100)$ & $5(31.2)$ & $11(68.8)$ & & $16(100)$ & $7(43.8)$ & $9(56.3)$ \\
\hline Non-PVTT $(n=34)$ & $4(11.8)$ & $30(88.2)$ & $18(52.9)$ & $15(44.1)$ & $5(14.7)$ & $29(85.3)$ & $19(55.9)$ & $14(41.2)$ \\
\hline$p$ value & $p=0.383$ & & $p=0.125$ & & $p=0.266$ & & $p=0.546$ & \\
\hline
\end{tabular}

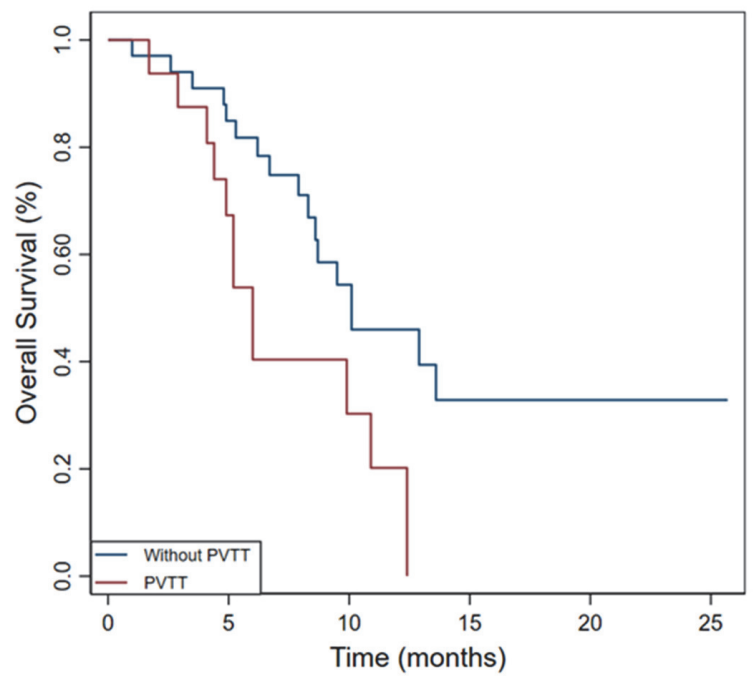

Abstract IDDF2020-ABS-0103 Figure 1 Significantly better overall survival rates were observed in the patients without portal vein tumor thrombus (PVTT) $(p=0.018)$

Results According to RECIST 1.1 criteria, no patient achieved a complete response (CR) while four (8\%) achieved partial response (PR); thus, the objective response rate (ORR) was $8 \%$. Nineteen (38\%) and $26(52 \%)$ patients exhibited stable disease (SD) and progressive disease (PD), respectively, at the first radiological assessment. The disease control rate (DCR) was $46 \%$ (table 1). The median OS was 9.5 months $(95 \%$ confidence interval [CI], 7.6-11.3), while the median TTP was 2.77 months (95\% CI, 2.1-3.5). In multivariate analysis, portal vein tumor thrombosis (PVTT) was an independent predictor of poor OS. Kaplan-Meier analysis revealed significantly shorter OS in the PVTT group than in the no PVTT group (median 6.0 vs. 10.1 months, $p=0.018$, (figure 1). HBV reactivation occurred in six patients (12\%), and the overall AEs rate was 92\%.

Conclusions PD-1 inhibitor may be safe and effective for HBV-related advanced HCC, with PVTT being a predictor of a poor prognosis.

\section{IDDF2020-ABS-0117 GUT MICROBIOTA ASSOCIATED WITH THE SENSITIVITY OF HEPATOCELLULAR CARCINOMA TO SORAFENIB}

${ }^{1}$ Weibin Lian*, ${ }^{2}$ Haiwei Li, ${ }^{3}$ Zhangran Chen, ${ }^{3}$ Yifan Lian. ${ }^{1}$ Quanzhou First Hospital Affiliated to Fujian Medical University, China; ${ }^{2}$ Liaoning Cancer Hospital and Institute, China; ${ }^{3}$ School of Medicine, Xiamen University, China

10.1136/gutjnl-2020-IDDF.154
Background Little is known about the relationship between alteration of gut microbiota and the sensitivity of hepatocellular carcinoma (HCC) to sorafenib. We performed a comparative study of gut microbiota composition between sorafenibresistant HCC patients ( $\mathrm{R}$ group, $\mathrm{n}=10$ ) and sorafenib sensitive HCC patients ( $\mathrm{S}$ group, $\mathrm{n}=10$ ).

Methods Twenty patients were classified into two groups based on the sensitivity of hepatocellular carcinoma to sorafenib within 12 months of post-sorafenib treatment. Treatment response was assessed using modified response evaluation criteria in solid tumors (mRECIST) criteria. After sorafenib treatment, the fecal samples were analyzed using 16S rRNA gene sequencing and LC-MS-based metabolomics approach.

Results Compared with the $\mathrm{R}$ group, significant gut microbiota alterations were associated with the sensitivity of HCC to sorafenib. The results showed that the $\mathrm{S}$ group had higher Faecalibacterium, Enterococcus and Veillonella abundance while the $\mathrm{R}$ group had higher levels of Lactobacillus and Prevotellaceae. Additionally, the S group had a higher bacterial network complexity compared with the $\mathrm{R}$ group. Moreover, both Salbutamol and Glyclopyramide correlated positively with Anaerostipes.

Conclusions These observations will lead to a better understanding of the relationship between alteration of gut microbiota and the sensitivity of HCC to sorafenib. Gut microbiota and microbe-associated metabolites can be used as diagnostic biomarkers in therapeutic explorations.

\section{IDDF2020-ABS-0139 GLOBAL BURDEN OF GALLBLADDER CANCER AND ITS ASSOCIATIONS WITH HDI, GDP, SMOKING, ALCOHOL DRINKING, AND OVERWEIGHT}

${ }^{1} J u n j i e$ Huang ${ }^{*},{ }^{2}$ Ping Chen, ${ }^{3}$ Shanjuan Wang, ${ }^{4}$ Lin Zhang, ${ }^{5}$ Jinqiu Yuan, ${ }^{1}$ Xiang-Qian Lao, ${ }^{1}$ Shelly LA Tse, ${ }^{6}$ Wanghong Xu, ${ }^{7}$ Zhi-Jie Zheng, 'Martin Wong. ${ }^{1} J o c k e y$ Club School of Public Health and Primary Care, Faculty of Medicine, Chinese University of Hong Kong, Hong Kong: ${ }^{2}$ Department of Gastroenterology, Ruijing Hospital North, School of Medicine, Shanghai Jiaotong University, China; ${ }^{3}$ Department of Gastroenterology, Jiading District Hospital, China; ${ }^{4}$ Melbourne School of Population and Global Health, The University of Melbourne, Australia; ${ }^{5}$ Clinical Research Centre; Scientific Research Centre, The Seventh Affiliated Hospital, Sun Yat-sen University, China; ${ }^{6}$ School of Public Health, Fudan University, China; 'Department of Global Health, School of Public Health, Peking University, China

\subsection{6/gutjpl-2020-IDDF.155}

Background This study aimed to evaluate the global incidence, mortality of gallbladder cancer, and their associations with human development index (HDI), gross domestic products (GDP), smoking, alcohol drinking, and overweight for 180 countries.

Methods The regional and national incidence and mortality figures for gallbladder cancer in 2018 were retrieved from the GLOBALCAN database. Age-standardized rates (ASRs) were evaluated by the Segi-Doll world standard population. HDI 